\title{
Parton-hadron duality in unpolarized and polarized structure functions
}

\author{
N. Bianchi* and A. Fantoni ${ }^{\dagger}$ \\ Laboratori Nazionali di Frascati dell'INFN, Via E. Fermi 40, 00044 Frascati (RM), Italy \\ S. Liuti ${ }^{\dagger}$ \\ University of Virginia, Charlottesville, Virginia 22901, USA \\ (Received 14 August 2003; published 28 January 2004)
}

\begin{abstract}
We study the phenomenon of parton-hadron duality in both polarized and unpolarized electron proton scattering using the HERMES and the Jefferson Lab data, respectively. In both cases we extend a systematic perturbative QCD based analysis to the integrals of the structure functions in the resonance region. After subtracting target mass corrections and large $x$ resummation effects, we extract the remaining power corrections up to order $1 / Q^{2}$. We find a sizable suppression of these terms with respect to analyses using deep inelastic scattering data. The suppression appears consistently in both polarized and unpolarized data, except for the low $Q^{2}$ polarized data, where a large negative higher twist contribution remains. A similar trend is found by using phenomenological parametrizations of the data, which include nonperturbative type corrections. Possible scenarios generating this behavior are discussed.
\end{abstract}

DOI: 10.1103/PhysRevD.69.014505

PACS number(s): $13.60 . \mathrm{Hb}, 13.88 .+\mathrm{e}$

\section{INTRODUCTION}

The structure of hadrons and their interactions can be described within two different but complementary approaches based on either partonic or hadronic degrees of freedom. The first one is expected to be valid at high energy, while the second one is applicable at low energy where the effects of confinement become large. In some specific cases where a description in terms of nonpartonic degrees of freedom seems more natural, the quark-gluon description can be also successfully used. This observation is called parton-hadron duality. It was introduced for deep inelastic scattering (DIS) by Bloom and Gilman [1] who reported an equivalence between the smooth $x$ dependence of the inclusive structure function at large $Q^{2}$ and the average over $W^{2}$ of the nucleon resonances $\left[x=Q^{2} / 2 M \nu, Q^{2}\right.$ is the four-momentum transfer squared, $M$ is the nucleon mass, $\nu$ is the energy transfer, and $W^{2}=Q^{2}(1 / x-1)+M^{2}$ is the final state invariant mass]. One refers to global duality if the average, defined, e.g., as the integral of the structure functions, is taken over the whole resonance region $1 \leqslant W^{2} \leqslant 4 \mathrm{GeV}^{2}$. If, however, the averaging is performed over smaller $W^{2}$ ranges, extending e.g. over single resonances, one can analyze the onset of local duality.

More generally, the concept of duality is often assumed in QCD-based interpretations of most hard scattering experiments, such as DIS, $e^{+} e^{-}$annihilation into hadrons, and hadron-hadron collisions. Its usage appears whenever hadronic observables (mostly averaged over a given energy range) are replaced by calculable partonic ones with little more going into the hadronic formation phase of each process-from partons to hadrons or vice versa. In a phenomenological context, duality studies are aimed at establishing to what extent a partonic description of the hard scat-

\footnotetext{
*Email address: bianchi@lnf.infn.it

†Email address: fantoni@lnf.infn.it

‡Email address: s14y@virginia.edu
}

tering process can determine the structure of the final state. In fact, as prescribed by the factorization property of QCD, we visualize hard scattering processes happening in two stages, one dominated by short times and distances and involving only parton jets, followed by hadron formation at a much larger scale. Duality is intrinsic to the factorization property. Violations of duality might signal violations of factorization in that, for instance, the probe-parton interaction might occur at larger time scales than required in order to exclude parton (re)interactions.

With the advent of both more detailed studies of soft scales and confinement [2], and higher precision measurements covering a wide range of reactions, it is now becoming possible to investigate the role of duality in QCD as a subject per se. For example, recent studies of local parton-hadron duality and its violations in semileptonic decays, and $\tau$ decays illustrate how the possible impact of these experiments on the extraction of the Cabibbo-Kobayashi-Maskawa (CKM) matrix elements, depends on the size of violations of local duality [3]. A practical necessity to address duality quantitatively exists also for inclusive $e p$ scattering where most of the currently available large $x$ data lie in the resonance region. In fact, for $x>0.5$ and $Q^{2} \geq 5 \mathrm{GeV}^{2}$ - a typical starting value for perturbative evolution- $-W^{2} \leqslant 5 \mathrm{GeV}^{2}$. Therefore, the behavior of the nucleon structure functions in the resonance region needs to be addressed in detail in order to be able to discuss theoretical predictions in the limit $x$ $\rightarrow 1$.

The first QCD-based studies of Bloom and Gilman duality reinterpreted the "averaging" procedure in terms of Mellin moments of the structure function. The moments taken in the low $Q^{2}$ and in the DIS regime, respectively, were shown to be equivalent to one another within the given range and precision of the data, modulo perturbative corrections and relatively small power corrections [4]. It was conjectured that duality resulted from a cancellation of higher order terms in the twist expansion that would otherwise be expected to dominate the cross section at $x \rightarrow 1$. This view has since been 
adopted, particularly in the more recent studies in Ref. [5]. In Ref. [6] a new analysis was performed, using the recent inclusive unpolarized electron-nucleon scattering data on hydrogen and deuterium targets from Jefferson Lab [7]. It was shown in particular that, because of the increased precision of the data, one is now able to unravel different sources of scaling violations affecting the structure functions, namely target mass corrections (TMC), large $x$ resummation effects (LxR), and dynamical higher twists (HTs), in addition to the standard next-to-leading-order (NLO) perturbative evolution. As a result, contrary to what was originally deduced in e.g. Ref. [7], a more pronounced role of the HT terms is obtained, pointing to the fact that duality, defined on the basis of a dominance of single parton scattering, could indeed be broken.

In contrast to the extensive study of duality for the unpolarized, i.e. spin averaged, photoabsorption cross section, the validity of duality has not been investigated until very recently for the spin structure function $g_{1}$, which is proportional to the spin-dependent photoabsorption cross section. Evidence of duality for the spin asymmetry $A_{1}$ was reported in Ref. [8]. A phenomenological study addressing partonduality was performed in [9] using the low $Q^{2}$ data from Ref. [10]. Studies of duality for $g_{1}$ are of particular interest because they might help understanding the transition from the large $Q^{2}$ regime described by $\mathrm{pQCD}$, and the $Q^{2} \rightarrow 0$ limit, where the Gerasimov-Drell-Hearn sum rule is expected to apply [11]. They may also lead to a complementary method to study the spin structure of the nucleon at large $x$, which is difficult to measure in the DIS region with high statistics. In particular, they might provide additional information on the transition from single parton scattering, to the dominance of processes where several partons are involved [12]. In this respect, it is important to perform an analysis aimed at disentangling the different contributions to the $Q^{2}$ dependence of $g_{1}$ in the resonance region. The aim of this paper is to carry out such an analysis by investigating quantitatively the onset of duality and its violations both for the unpolarized and polarized structure functions.

In Sec. II we define the concept of duality and we illustrate the role of different kinematical regions; in Sec. III we present our analysis and we describe our results. In particular, we compare the data with perturbative-QCD predictions and we discuss in detail the contribution of different types of corrections in both the unpolarized and polarized case. Finally, in Sec. IV we draw our conclusions.

\section{DEFINITIONS AND KINEMATICS}

Parton-hadron duality in DIS was first observed more than three decades ago. Since then it has been necessary to give new definitions of the quantities involved which can be described within QCD-based approaches. In what follows we list all such definitions.

\section{A. Kinematical variables}

Besides the scaling variable $x$, other variables have been used in the literature to study duality. A number of param-

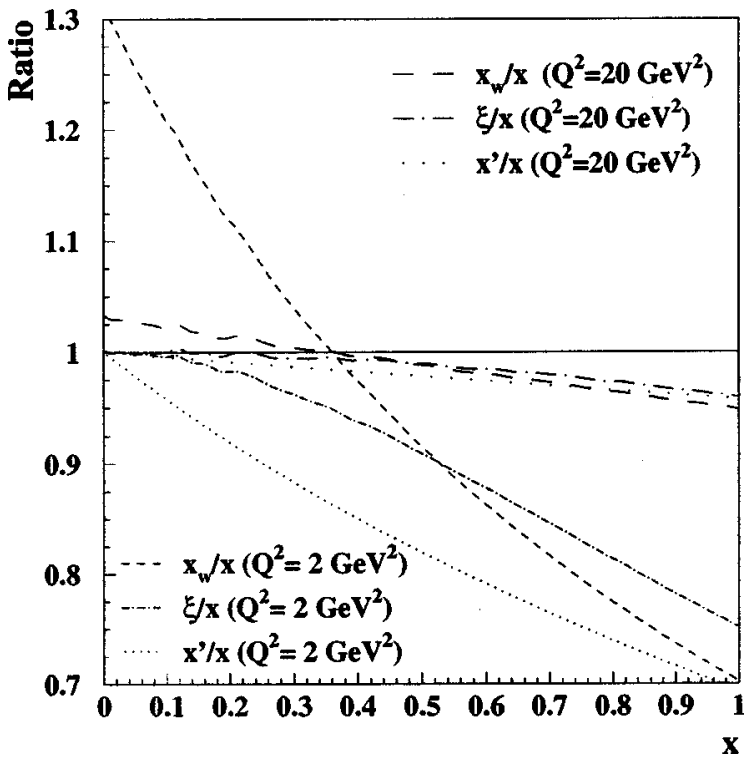

FIG. 1. Ratio between the three different variables $x^{\prime}, \xi$ and $x_{W}$ defined in the text and the Bjorken variable $x$ as a function of $x$.

etrizations based on these variables have been proposed that reproduce in an effective way some of the corrections to the perturbative QCD calculations that we study in this paper. The most extensively used variables are $x^{\prime}=1 / \omega^{\prime}$, where $\omega^{\prime}=1 / x+M^{2} / Q^{2}$ ( $x^{\prime}$ was originally introduced by Bloom and Gilman in order to obtain a better agreement between DIS and the resonance region); $\xi=2 x /[1+(1$ $\left.+4 x^{2} M^{2} / Q^{2}\right)^{1 / 2}$ ] [13], originally introduced to take into account the target mass effects; $x_{w}=Q^{2}+B /\left(Q^{2}+W^{2}-M^{2}\right.$ $+A), A$ and $B$ being fitted parameters, used in Refs. $[14,15]$. These additional variables include a $Q^{2}$ dependence that phenomenologically absorbs some of the scaling violations that are important at low $Q^{2}$. In Fig. 1 we compare their behavior vs $x$ for different values of $Q^{2}$. From the figure one can see that by calculating $F_{2}$ in $\xi$ and $x^{\prime}$, one effectively "rescales" the structure function to lower values of $x$, in a $Q^{2}$ dependent way, namely the rescaling is larger at lower $Q^{2}$.

In this paper we present results in terms of $x$ and $Q^{2}$ and we illustrate the contributions of scaling violations of different origin on a case by case basis.

\section{B. Unpolarized structure function}

The inclusive DIS cross section of unpolarized electrons off an unpolarized proton is written in terms of the two structure functions $F_{2}$ and $F_{1}$,

$$
\frac{d^{2} \sigma}{d x d y}=\frac{4 \pi \alpha^{2}}{Q^{2} x y}\left[\left(1-y-\frac{(M x y)^{2}}{Q^{2}}\right) F_{2}+y^{2} x F_{1}\right],
$$

where $y=\nu / E, E$ being the initial electron energy. The structure functions are related by the equation

$$
F_{1}=F_{2}\left(1+\gamma^{2}\right) /[2 x(1+R)],
$$

where $\gamma^{2}=4 M^{2} x^{2} / Q^{2}$, and $R$ is the ratio of the longitudinal to the transverse virtual photo-absorption cross sections. 
In QCD, $F_{2}$ is expanded in series of inverse powers of $Q^{2}$, obtained by ordering the matrix elements in the DIS process by increasing twist $\tau$, which is equal to their dimension minus spin,

$$
F_{2}\left(x, Q^{2}\right)=F_{2}^{L T}\left(x, Q^{2}\right)+\frac{H\left(x, Q^{2}\right)}{Q^{2}}+\mathcal{O}\left(1 / Q^{4}\right) .
$$

The first term is the leading twist (LT), with $\tau=2$. The terms of order $1 / Q^{\tau-2}, \tau \geqslant 4$, in Eq. (3) are the higher order terms, generally referred to as higher twists. Additional corrections to the LT part due to the finite mass of the initial nucleonthe target mass corrections (TMC) - are included directly in $F_{2}^{L T}$. For $Q^{2}$ larger than $\approx 1 \mathrm{GeV}^{2}, \mathrm{TMC}$ are taken into account through the following expansion [16]:

$$
\begin{aligned}
F_{2}^{L T(T M C)}\left(x, Q^{2}\right)= & \frac{x^{2}}{\xi^{2} \gamma^{3}} F_{2}^{\infty}\left(\xi, Q^{2}\right) \\
& +6 \frac{x^{3} M^{2}}{Q^{2} \gamma^{4}} \int_{\xi}^{1} \frac{d \xi^{\prime}}{\xi^{\prime 2}} F_{2}^{\infty}\left(\xi^{\prime}, Q^{2}\right),
\end{aligned}
$$

where $F_{2}^{\infty}$ is the structure function in the absence of TMC. Following the original suggestion of [17], only terms up to order $M^{2} / Q^{2}$, i.e. of the same order of the HT term extracted from Eq. (3), are kept in the expansion, so as to minimize ambiguities in the behavior of $F_{2}$ at $x \approx 1$.

$H$, then, represents the "genuine" HT correction that involves interactions between the struck parton and the spectators or, formally, multiparton correlation functions, $F_{2}$.

Parton-hadron duality in DIS is studied by considering integrals of the structure function defined as

$$
I^{\mathrm{res}}\left(Q^{2}\right)=\int_{x_{\min }}^{x_{\max }} F_{2}^{\mathrm{res}}\left(x, Q^{2}\right) d x
$$

where $F_{2}^{\text {res }}$ is evaluated using the experimental data in the resonance region. For each $Q^{2}$ value, $x_{\min }=Q^{2} /\left(Q^{2}+W_{\max }^{2}\right.$ $\left.-M^{2}\right)$, and $x_{\max }=Q^{2} /\left(Q^{2}+W_{\min }^{2}-M^{2}\right)$. $W_{\min }$ and $W_{\max }$ delimit the resonance region. The same expression is then calculated in the same range of $x$ and for the same value of $Q^{2}$, using parametrizations of $F_{2}$ that reproduce the DIS behavior of the data at large $Q^{2}$. These parametrizations are very well constrained in the region of interest $(x>0.3)$ although they do not correspond directly to measured data. Here in fact $F_{2}$ is dominated by the valence contribution. On the contrary, by using the same procedure at low $x$ where the singlet and gluon distributions govern $F_{2}$, one would find much larger uncertainties in the initial low $Q^{2}$ parametrizations because of their strong correlation with the value of $\alpha_{S}$. We present two forms for the DIS integrals:

$$
I^{L T}\left(Q^{2}\right)=\int_{x_{\min }}^{x_{\max }} F_{2}^{L T}\left(x, Q^{2}\right) d x,
$$

and

$$
I^{H T}\left(Q^{2}\right)=\int_{x_{\min }}^{x_{\max }}\left(F_{2}^{L T}\left(x, Q^{2}\right)+\frac{H\left(x, Q^{2}\right)}{Q^{2}}\right) d x .
$$

Duality is attained strictly only if the ratio

$$
R_{\text {unpol }}^{L T}=\frac{I^{\text {res }}}{I^{L T}}
$$

is unity. However, one can extend this definition to the ratio

$$
R_{\mathrm{unpol}}^{H T}=\frac{I^{\mathrm{res}}}{I^{H T}},
$$

assuming that the HT contribution to $I_{H T}$ is small, in accordance with the twist expansion, Eq. (3).

Perturbative QCD analyses use the Mellin moments of the structure function, which allow for a direct comparison with theoretical predictions. These are defined as

$$
M_{n}\left(Q^{2}\right)=\int_{0}^{1} d x x^{n-2} F_{2}\left(x, Q^{2}\right),
$$

and by

$$
\begin{aligned}
M_{n}^{T M C}\left(Q^{2}\right)= & \int_{0}^{1} d x \xi^{n-1} \frac{F_{2}\left(x, Q^{2}\right)}{x} p_{n}\left(\frac{\xi}{x}\right), \\
p_{n}= & 1+\frac{6(n-1)}{(n+2)(n+3)}\left(\frac{\xi}{x}-1\right) \\
& +\frac{n(n-1)}{(n+2)(n+3)}\left(\frac{\xi}{x}-1\right)^{2},
\end{aligned}
$$

which takes into account TMC [13]. However, one needs in this case experimental values of the structure function in kinematics outside the resonance region. This procedure renders the comparison between theory and experiment less straightforward. The difference between the Mellin moments [Eqs. (9), (11)] and the integrals over the resonance region$I_{n}=\int_{x_{\min }}^{x_{\max }} x^{n-2} F_{2}^{L T}\left(x, Q^{2}\right) \mathrm{dx}$-is shown in Fig. 2. The drop of the quantities $I_{n}$ with respect to $M_{n}$ at larger values of $Q^{2}$, is due to the pQCD evolution of $F_{2}$, that moves strength to lower values of $x$, outside the range $\left[x_{\max }, x_{\min }\right]$. In our approach we use the integrals defined in Eqs. (5), (6). As it can be understood also from the trend shown in Fig. 2, these effectively describe duality also as a function of the average value of $x$ in each interval $\left[x_{\min }\left(Q^{2}\right), x_{\max }\left(Q^{2}\right)\right]$. This corresponds to $\langle x\rangle=x\left(W^{2} \equiv 2.5 \mathrm{GeV}^{2}\right)$.

It is also possible to consider a third approach, namely a point by point comparison of $F_{2}$ both in the DIS and in the resonance region [6]. The latter can in fact be fitted to a smooth curve tracing the resonances with a very high accuracy given by the increased precision of the new Jefferson Lab measurements. They are then compared directly to DIS parametrizations $F_{2}$ at the same $x$ and $Q^{2}$ values.

We notice that the approach using moments includes the contribution from elastic $e p$ scattering entering the integrals 


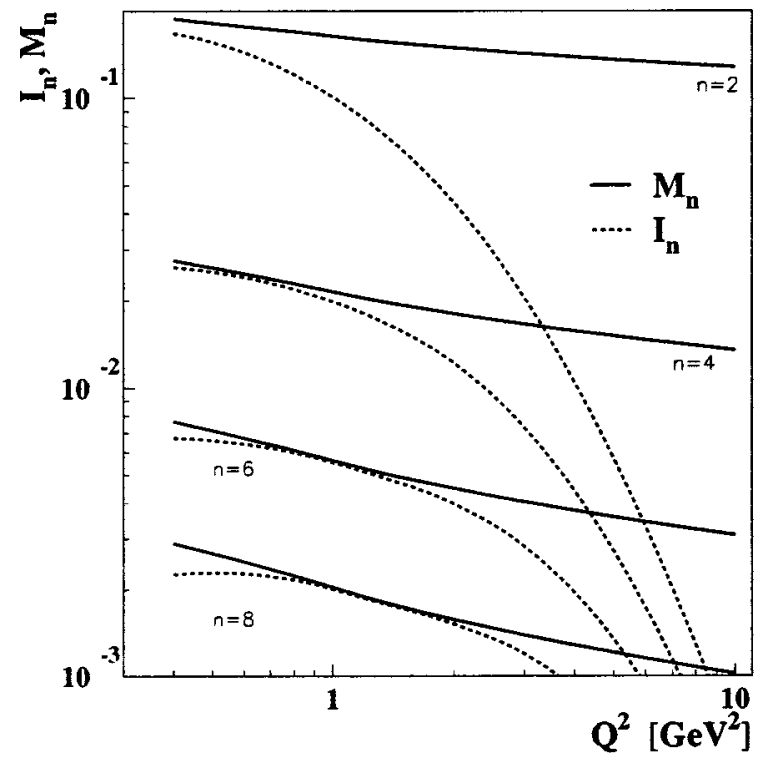

FIG. 2. The integral $I_{n}$ defined in the text plotted vs $Q^{2}$ (dashed line), compared to the Mellin moments defined in Eq. (9) (full lines). All quantities have been calculated for illustration using the parton distributions function parametrization from Ref. [25].

in Eqs. (9), (11) at $x=1$. Furthermore, as shown in Ref. [18], the elastic contribution governs the moments' behavior at $Q^{2} \lesssim 1 \mathrm{GeV}^{2}$. In the procedure described here, as well as in the point by point comparison of Ref. [6], the elastic contribution is instead manifestly excluded by the kinematics. Our approach renders therefore a better description of the resonances' role at low $Q^{2}$. A combination of the three types of analyses described in $[6,18]$ and in the present paper, is necessary in order to obtain a quantitative interpretation of the $Q^{2}$ dependence of duality and of its violation.

\section{Polarized structure function}

The spin-dependent part of the polarized deep inelastic cross section is given by

$$
\begin{aligned}
\frac{d^{2} \sigma}{d x d y}= & \frac{e^{4}}{2 \pi^{2} Q^{2}}\left(-H_{e} H_{N}\right) \\
& \times\left[\left(1-\frac{y}{2}-\frac{y^{2}}{4} \gamma^{2}\right) g_{1}\left(x, Q^{2}\right)-\frac{y}{2} \gamma^{2} g_{2}\left(x, Q^{2}\right)\right],
\end{aligned}
$$

where $H_{e}$ and $H_{N}$ are the polarizations of the incident electron and of the nucleon of the target, respectively.

Because of the mixing of $g_{1}$ and $g_{2}$, a precise determination of $g_{1}$ from a longitudinally polarized target alone is not possible. The experimentally measured cross section asymmetries are the longitudinal $A_{\|}$and the transverse $A_{\perp}$ ones, formed from combining data with opposite beam helicity:

$$
A_{\|}=\frac{\sigma^{\downarrow \uparrow}-\sigma^{\uparrow \uparrow}}{\sigma^{\downarrow \uparrow}+\sigma^{\uparrow \uparrow}}, \quad A_{\perp}=\frac{\sigma^{\downarrow \rightarrow}-\sigma^{\uparrow \rightarrow}}{\sigma^{\downarrow \rightarrow}+\sigma^{\uparrow \rightarrow}} .
$$

The polarized structure functions are determined from these asymmetries:

$$
\begin{aligned}
& g_{1}\left(x, Q^{2}\right)=\frac{F_{1}\left(x, Q^{2}\right)}{d^{\prime}}\left[A_{\|}+\tan \theta / 2 \cdot A_{\perp}\right], \\
& g_{2}\left(x, Q^{2}\right)=\frac{y F_{1}\left(x, Q^{2}\right)}{2 d^{\prime}}\left[\frac{E+E^{\prime} \cos \theta}{E^{\prime} \sin \theta} A_{\perp}-A_{\|}\right]
\end{aligned}
$$

where $E^{\prime}$ is the scattered lepton energy, $\theta$ is the scattering angle, $d^{\prime}=[(1-\epsilon)(2-y)] /\left[y\left(1+\epsilon R\left(x, Q^{2}\right)\right]\right.$, with $\epsilon$ being the degree of transverse polarization of the virtual photon and defined as $\epsilon^{-1}=1+2\left(1+\gamma^{-2}\right) \tan ^{2}(\theta / 2)$.

The virtual photon-absorption asymmetries $A_{1}$ and $A_{2}$ are related to the measured asymmetries by

$$
\begin{aligned}
& A_{\|}=D\left(A_{1}+\eta A_{2}\right) \\
& A_{\perp}=d\left(A_{2}-\zeta A_{1}\right),
\end{aligned}
$$

where $D$ is the depolarization factor $D=y(2-y)(1$ $\left.+\gamma^{2} y / 2\right) /\left[y^{2}\left(1+\gamma^{2}\right)\left(1-2 m_{e}^{2} / Q^{2}\right)+2\left(1-y-\gamma^{2} y^{2} / 4\right)(1\right.$ $+R)]$, and $d=D \sqrt{2 \epsilon /(1+\epsilon)}, \eta=2 \gamma(1-y) /(2-y)$ and $\zeta$ $=\eta(1+\epsilon) / 2 \epsilon$ are kinematic factors.

From the measured asymmetries $A_{\|}$and $A_{\perp}$, the virtual photon asymmetries can be related to the photon absorption cross section of the nucleon for a given $x$ and $Q^{2}$ :

$$
\begin{gathered}
A_{1}=\frac{\sigma_{1 / 2}-\sigma_{3 / 2}}{\sigma_{1 / 2}+\sigma_{3 / 2}}=\frac{\sigma_{T T}}{\sigma_{T}}=\frac{g_{1}-\gamma^{2} g_{2}}{F_{1}} \\
A_{2}=\frac{2 \sigma_{L T}}{\sigma_{1 / 2}+\sigma_{3 / 2}}=\frac{\sigma_{L T}}{\sigma_{T}}=\frac{\gamma\left(g_{1}+g_{2}\right)}{F_{1}} .
\end{gathered}
$$

Here $\sigma_{1 / 2}$ and $\sigma_{3 / 2}$ are the virtual photoabsorption cross section when the projection of the angular momentum of the photon-nucleon system along the incident photon direction is $1 / 2$ or $3 / 2$ respectively, $\sigma_{L T}$ is the interference term between the transverse and longitudinal photon-nucleon amplitudes, respectively given by $\sigma_{T}=\left(\sigma_{1 / 2}+\sigma_{3 / 2}\right) / 2$ and $\sigma_{T T}=\left(\sigma_{1 / 2}\right.$ $\left.-\sigma_{3 / 2}\right) / 2$. If only the longitudinal asymmetry is measured, it is necessary to make an assumption for the asymmetry $A_{2}$.

From the measured asymmetry it is possible to evaluate the polarized structure function $g_{1}$ :

$$
g_{1}\left(x, Q^{2}\right) \approx A_{1}(x) F_{1}\left(x, Q^{2}\right) .
$$

Equation (17) was obtained by neglecting the term $\gamma^{2} g_{2}$ in Eq. (16). This is an adequate approximation for describing the data within their given accuracy. In fact, $\gamma^{2} g_{2}$ vanishes in the DIS limit. In the range of data considered, $\gamma^{2}$ is larger$\gamma^{2} \approx 0.44$ at $Q^{2}=0.5 \mathrm{GeV}^{2}$, and $\gamma^{2} \approx 0.36$ at $Q^{2}$ $=5.4 \mathrm{GeV}^{2}$. However, the Burkhardt-Cottingham sum rule, $\int_{0}^{1} g_{2}\left(x, Q^{2}\right) d x=0$ [19], is believed to hold. This ensures the smallness of the neglected term, barring a strong oscillating behavior in $x$ of $g_{2}$.

As for the unpolarized case, the twist expansion reads 


$$
g_{1}\left(x, Q^{2}\right)=g_{1}^{L T}\left(x, Q^{2}\right)+\frac{\widetilde{H}\left(x, Q^{2}\right)}{Q^{2}}+\mathcal{O}\left(1 / Q^{4}\right),
$$

where, using Eqs. (2), (4), (17),

$$
\begin{aligned}
g_{1}^{L T}\left(x, Q^{2}\right)= & F_{2}^{L T}\left(x, Q^{2}\right) A_{1}^{e x p}(x)\left(1+\gamma^{2}\right) / \\
& {\left[2 x\left(1+R^{e x p}\right)\right], }
\end{aligned}
$$

the superscript "exp" emphasizing that we have used the experimental values for the quantities under consideration. Notice that HT contributions that could in principle appear on the r.h.s. of Eq. (19) through $A_{1}^{\text {exp }}$ and $R^{\text {exp }}$, are negligible. In fact, the asymmetry $A_{1}^{\exp }$ was found not to depend on $Q^{2}$ (see [8] and discussion below). Moreover, the impact of $R^{\exp }$ on the evaluation of $g_{1}$ is of the order of a few percent, well below the accuracy of current polarized data.

We study parton-hadron duality by defining the integrals

$$
\widetilde{\Gamma}_{1}^{\mathrm{res}}=\int_{x_{\min }}^{x_{\max }} g_{1}^{\mathrm{res}}\left(x, Q^{2}\right) d x,
$$

where $g_{1}^{\text {res }}$ is obtained from the data in the resonance region, and

$$
\begin{gathered}
\Gamma_{1}^{L T}=\int_{x_{\min }}^{x_{\max }} g_{1}^{L T}\left(x, Q^{2}\right) d x \\
\widetilde{\Gamma}_{1}^{H T}=\int_{x_{\min }}^{x_{\max }}\left(g_{1}^{L T}\left(x, Q^{2}\right)+\frac{\widetilde{H}\left(x, Q^{2}\right)}{Q^{2}}\right) d x .
\end{gathered}
$$

The ratios are given by

$$
R_{\mathrm{pol}}^{L T}=\frac{\widetilde{\Gamma}_{1}^{\mathrm{res}}}{\widetilde{\Gamma}_{1}^{L T}}, \quad R_{\mathrm{pol}}^{H T}=\frac{\widetilde{\Gamma}_{1}^{\mathrm{res}}}{\widetilde{\Gamma}_{1}^{H T}} .
$$

As for the unpolarized case, duality is verified if either ratio is unity.

\section{ANALYSIS AND INTERPRETATION OF DATA}

In this section we present a quantitative analysis of the $Q^{2}$ dependence of parton-hadron duality in both polarized and unpolarized $e p$ scattering. We take into account all current data in the resonance region, $1 \leqslant W^{2} \leqslant 4 \mathrm{GeV}^{2}$. For the unpolarized case we used data obtained at Jefferson Lab in the range $0.3 \leqslant Q^{2} \leqslant 5 \mathrm{GeV}^{2}$ [7], and data from SLAC ([20] and references therein) for $Q^{2} \geqslant 4 \mathrm{GeV}^{2}$. For the polarized case there are only a few experimental data in the resonance region. One set is part of the E143 data [10], and it corresponds to $Q^{2}=0.5$ and $1.2 \mathrm{GeV}^{2}$. Another set is the one from HERMES $[8,21]$ in the range $1.2 \leqslant Q^{2} \leqslant 12 \mathrm{GeV}^{2}$.

In the polarized case the $Q^{2}$ dependence originates from the structure function $F_{1}$ and from the ratio $R$. In the evaluation of the denominators of Eqs. (22), we used the SLAC global analysis [22] parametrization for $R$ and, for $A_{1}$, a power law fit to the world DIS data at $x>0.3: A_{1}=x^{0.7}$, as already shown in Ref. [8]. This parametrization of $A_{1}$ is con- strained to 1 at $x=1$ and it does not depend on $Q^{2}$, as indicated by experimental data in this range [23].

\section{A. Comparison with pQCD}

The unpolarized structure function $F_{2}$ was first evaluated from dynamical parametrizations, coming from the parton distribution functions (PDFs): MRST [24], CTEQ [25], GRV94 [26] and GRV98 [27]. The last two parametrizations have been evaluated at leading order (LO) and at next to leading order (NLO). All of them are pure DIS parametrizations and they were extended to the measured $x$ and $Q^{2}$ ranges by $\mathrm{pQCD}$ evolution. The $Q^{2}$ evolution of the polarized parton densities is governed by the Dokshitzer-GribovLipatov-Altarelli-Parisi (DGLAP) [28] equations. The results are shown in Fig. 3, in the top panel for the unpolarized data and in the bottom panel for the polarized data. The uncertainty due to the use of different LO and NLO parametrizations is represented by a band labeled "PDF uncertainty." A potential theoretical error in the extrapolation of the ratios to low $Q^{2}$ could be generated by the error in $\alpha_{S}\left(M_{Z}^{2}\right)$. However, because the structure functions are dominated by large $x$ kinematics, DGLAP evolution proceeds only through nonsinglet (NS) distributions. This explains why there is very little uncertainty in the extrapolation of the initial pQCD distribution to the low values of $W^{2}$ considered, and also the small difference between LO and NLO evolution. The other band in the figure represents the experimental error, calculated as the sum in quadrature of the statistical and systematic errors of the data in the resonance region.

Parton-hadron duality is not fulfilled by using solely the PDFs up to NLO in both the unpolarized and polarized structure functions $F_{2}$ and $g_{1}$. However it is possible to see a different behavior between $R_{\text {unpol }}$ and $R_{\text {pol }}$. In the unpolarized case the ratio is increasing with $Q^{2}$, but for the polarized case the situation is different: while at low $Q^{2}$ the ratio is significantly below unity and shows a strong increase with $Q^{2}$, at higher $Q^{2}$ the ratio derived from HERMES is above unity and it appears to be weakly dependent on $Q^{2}$ within error bars.

In Fig. 4 we further illustrate the origin of this behavior by plotting separately the numerator (data points) and the denominator ("theoretical" curves representing a pQCD based parametrization), of the ratios $R_{\text {unpol }}$ and $R_{\text {pol }}$, respectively. We also plot the integral of $F_{1}$ (dotted line) in order to show the effect of both $A_{1}$ and of the $Q^{2}$-dependent factors that come into play in the definition of $g_{1}$. All quantities are plotted vs $x \equiv\langle x\rangle$, i.e. the average value of Bjorken $x$ for each spectrum, defined in Sec. II. Notice that the value of $\langle x\rangle$ increases with $Q^{2}$. The trends in the figure suggest therefore that similarly to what was observed in DIS, in the resonance region there are corrections beyond DGLAP evolution that are positive at large $x$, and negative at smaller $x$, the threshold being defined by $x \approx 0.33-0.43$ and $Q^{2} \approx 1 \mathrm{GeV}^{2}$. However, while these corrections are comparable in size for both the polarized and unpolarized case at large $x$, at low $x$ there seems to be a much larger nonperturbative effect for the polarized data. While possible explanations have been suggested e.g. in [29], it is clear that more data in this region 

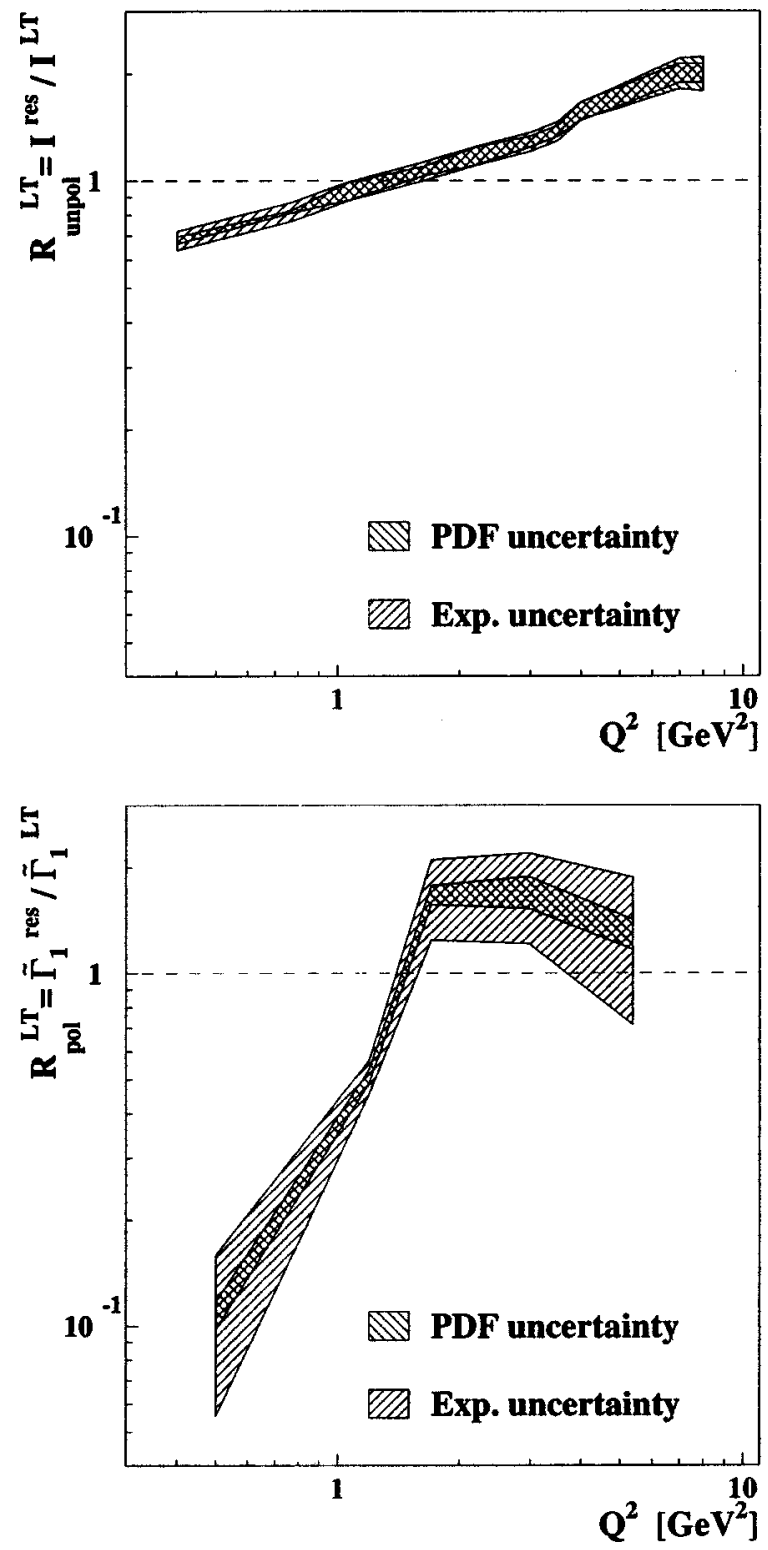

FIG. 3. Ratio between the integral of the structure function as measured in the resonance region and as parametrized in the DIS region, as a function of $Q^{2}$. The top panel refers to the unpolarized case, while the bottom panel to the polarized one. One band represents the theoretical uncertainty due to the use of different LO and NLO parametrizations: MRST [24], CTEQ [25], GRV94 [26], GRV98 [27]. The other band represents the experimental uncertainty, that is the sum in quadrature of the statistical and systematic uncertainties of the data in the resonance region.

would help in disentangling the $Q^{2}$ dependence and the possible size of the nonperturbative effects.

\section{B. Comparison with phenomenological parametrizations}

The unpolarized structure function $F_{2}$ has been evaluated from three different phenomenological fits to DIS data $[15,30,31]$ and scaled to the same $Q^{2}$ values as for $I^{\text {res }}$ and $\widetilde{\Gamma}_{1}^{\text {res }}$ to take into account the large effect of scaling violation at large $x$.

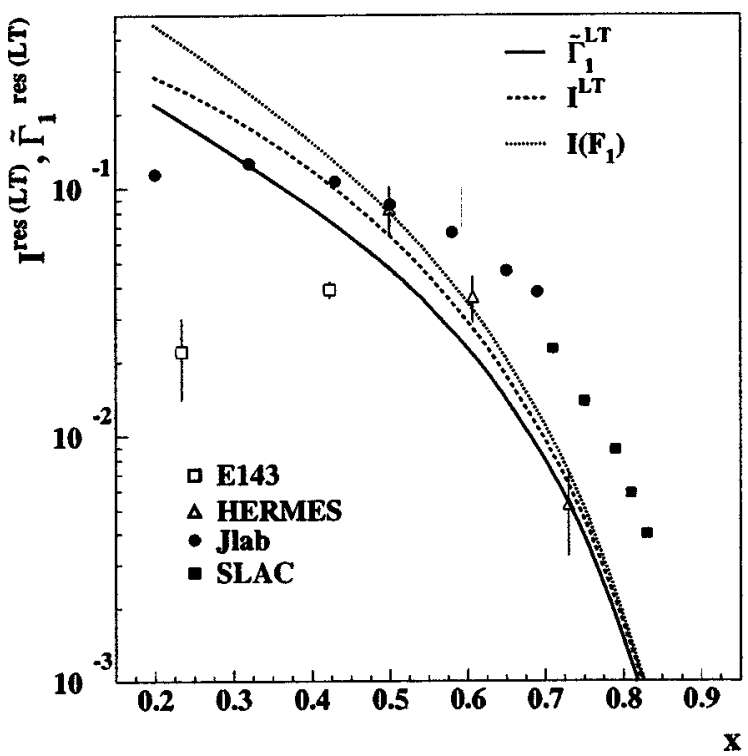

FIG. 4. The integrals $I^{\text {res }}$, Eq. (4), and $\widetilde{\Gamma}_{1}^{\text {res }}$, Eq. (4) plotted vs the average value of Bjorken $\mathrm{x}$ defined in the text, obtained using the data from Refs. $[8,10]$. Experimental data are compared to the integrals of the valence component of the structure functions $g_{1}$ (full line), $F_{2}$ (dashed line), and $F_{1}$ (dotted line), calculated using NLO parametrizations.

The parametrization from Ref. [15] is a modification of the PDF [26] that purports to include target mass effects through an effective change of variable, higher twist effects at high $x$, and a factor that enables an extension of the fit down to the photoproduction limit. The ALLM parametrization [30] is based on a reggeon and pomeron exchange and it was constructed for the high $W^{2}$ limit. We included it because it can be extended to very low $Q^{2}$ values. The NMC parametrization [31] includes a fit of $H T$ terms using world data with $W^{2}>10 \mathrm{GeV}^{2}$.

The ratios $R_{\text {unpol }}^{\mathrm{DIS}}=I^{\mathrm{res}} / I^{\mathrm{DIS}}$ and $R_{\mathrm{pol}}^{\mathrm{DIS}}=\widetilde{\Gamma}_{1}^{\mathrm{res}} / \widetilde{\Gamma}_{1}^{\mathrm{DIS}}$, where $I^{\mathrm{DIS}}$ and $\widetilde{\Gamma}_{1}^{\mathrm{DIS}}$ are the integrals calculated with these phenomenological parametrizations, are shown in Fig. 5 in the top and bottom panel, respectively, for several $Q^{2}$-values.

In the unpolarized case the slope of the ratio is less evident compared to the previous method shown in Fig. 3 and the ratio is well consistent with unity for $Q^{2}>2 \mathrm{GeV}^{2}$. In the polarized case, the ratio at higher $Q^{2}$ derived from HERMES data is consistent with unity inside the experimental errors and still independent of $Q^{2}$. However, at low $Q^{2}$ the uncertainty on the parametrization is bigger than what was found from the PDFs.

Since these phenomenological parametrizations are obtained by fitting deep-inelastic data even in the low $Q^{2}$ region, they can implicitly include nonperturbative effects and this may explain the "observation of duality."

\section{Size of nonperturbative contributions}

In order to understand the nature of the remaining $Q^{2}$ dependence that cannot be described by NLO pQCD evolution, we studied the effect of TMC and LxR on the ratios $R_{\text {unpol }}^{H T}$ and $R_{p o l}^{H T}$. The analysis was performed by using $x$ as 

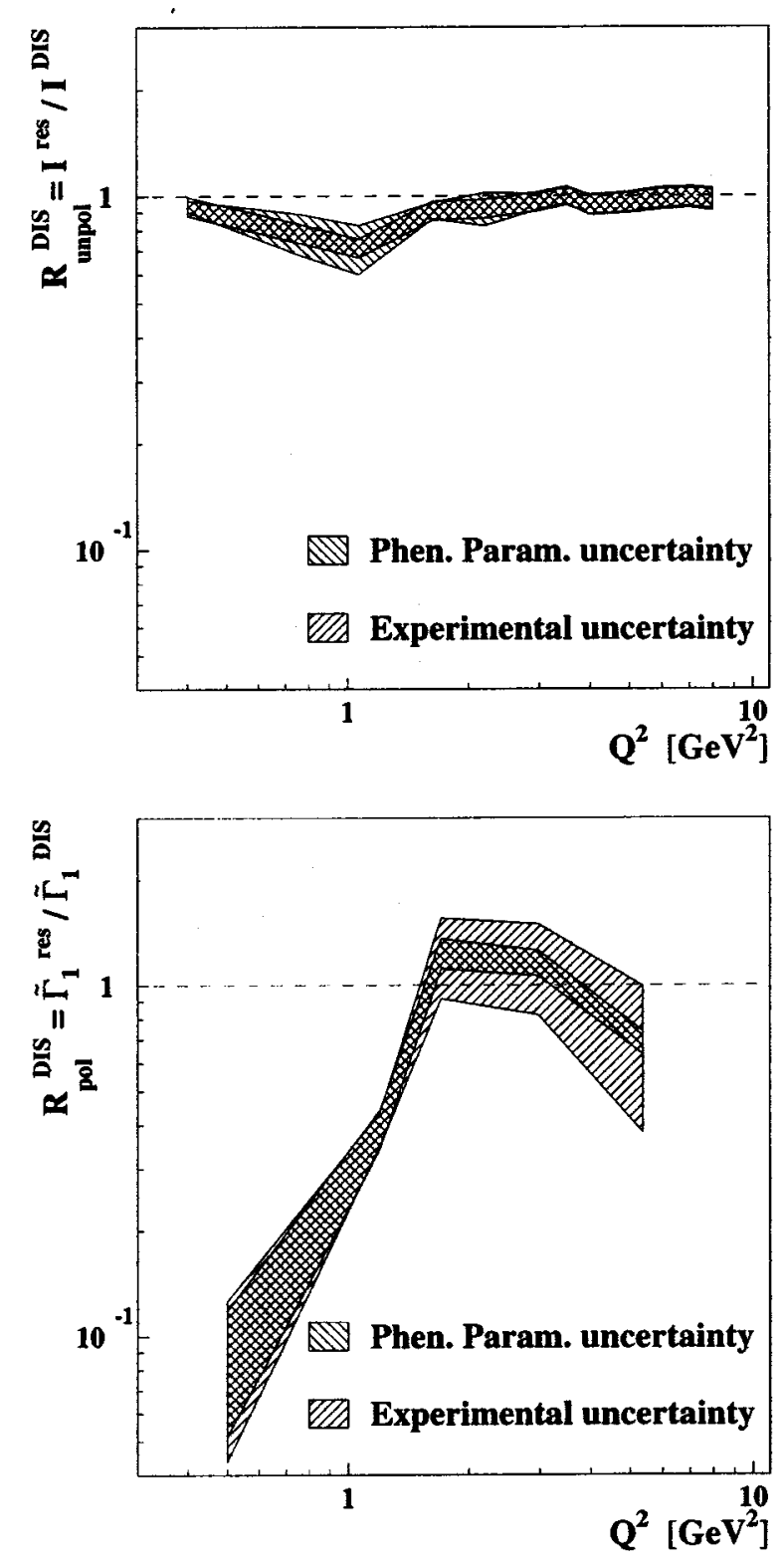

FIG. 5. Ratio between the integral of the structure function measured in the resonance region and as parametrized in the DIS region $[15,30,31]$, as a function of $Q^{2}$. The top panel refers to the unpolarized case, while the bottom panel to the polarized one. Notations are the same as in Fig. 3.

an integration variable, which avoids the ambiguities associated to the usage of other ad hoc kinematical variables. We used standard input parametrizations with initial scale $Q_{o}^{2}$ $=1 \mathrm{GeV}^{2}$. Once TMC and LxR have been subtracted from the data, and assuming the validity of the twist expansion, Eqs. (3), (18) in this region, one can interpret any remaining discrepancy in terms of HTs.

We notice that although we did not consider NNLO calculations, these are not expected to alter substantially our extraction since, differently from what was seen originally in the case of $F_{3}$, these have been proven to give a relatively small contribution to $F_{2}$ [32].

The value of $\alpha_{S}\left(M_{Z}^{2}\right)$ that was used in our calculations corresponds to the one given for the DIS parametrizations. It has been long noticed that a correlation exists between $\alpha_{S}$ and the extracted values of the HTs (see [33] and references therein and the recent highly accurate determination in Ref. [34]). It is exactly because of this correlation that we keep its value fixed from evaluations in a region where the HTs contribution is negligible. This statement is equivalent to saying that $\alpha_{S}$ cannot be extracted reliably from large $x$ data.

TMC have been evaluated using Eq. (4) for the unpolarized case, and Eqs. (4), (19) for the polarized data. Although this procedure disregards parton off-shell effects that might be important in the resonance region (see Refs. [35,36]), we emphasize here its power expansion character, and we set as a limiting condition for its validity, that the inequality $x^{2} M^{2} / Q^{2}<1$ be verified [6]. Therefore, current treatments of TMC in the resonance region are uncertain for values of $Q^{2} \lesssim 1.5 \mathrm{GeV}^{2}$.

LxR effects arise formally from terms containing powers of $\ln (1-z), z$ being the longitudinal variable in the evolution equations, that are present in the Wilson coefficient functions $C(z)$. The latter relate the parton distributions to e.g. the structure function $F_{2}$, according to

$$
F_{2}^{N S}\left(x, Q^{2}\right)=\frac{\alpha_{s}}{2 \pi} \sum_{q} \int_{x}^{1} d z C_{N S}(z) q_{N S}\left(x / z, Q^{2}\right),
$$

where we have considered only the nonsinglet (NS) contribution to $F_{2}$ since only valence quarks distributions are relevant in our kinematics. The logarithmic terms in $C_{N S}(z)$ become very large at large $x$, and they need to be resummed to all orders in $\alpha_{S}$. This can be accomplished by noticing that the correct kinematical variable that determines the phase space for the radiation of gluons at large $x$, is $\widetilde{W}^{2}$ $=Q^{2}(1-z) / z$, instead of $Q^{2}[37,38]$. As a result, the argument of the strong coupling constant becomes $z$-dependent: $\alpha_{S}\left(Q^{2}\right) \rightarrow \alpha_{S}\left[Q^{2}(1-z) / z\right]$ (see [39] and references therein). In this procedure, however, an ambiguity is introduced, related to the need of continuing the value of $\alpha_{S}$ for low values of its argument, i.e. for $z$ very close to 1 [40]. The size of this ambiguity could be of the same order of the HT corrections. Nevertheless, our evaluation is largely free from this problem because of the particular kinematical conditions in the resonance region. We are in fact studying the structure functions at fixed $W^{2}$, in between $1 \leqslant W^{2} \leqslant 4 \mathrm{GeV}^{2}$. Consequently $Q^{2}$ increases with $x$. This softens the ambiguity in $\alpha_{S}$, and renders our procedure reliable for the extraction of HT terms. We illustrate this situation in Fig. 6 where we plot the value of $\alpha_{S}$ at $Q^{2}=10 \mathrm{GeV}^{2}$, and we compare it with the resummed value in the resonance region, at $Q^{2}(1-z) / z$ for a fixed average $Q^{2}$, and at $Q^{2}(x)(1-z) / z$, with $Q^{2}(x)$ $=W^{2}\langle x\rangle /(1-\langle x\rangle)$, and $\langle x\rangle=0.83$.

All of the effects described in this section are summarized in the upper panel of Fig. 7. In the figure we plot the ratio $R_{\text {unpol }}^{L T}$, from Eq. (7), where the numerator is obtained from the experimental data, while the denominator includes the different components of our analysis, one by one. For unpolarized scattering we find that TMC and LxR diminish considerably the space left for HT contributions. The contribution of TMC is large at the largest values of $Q^{2}$ because these 


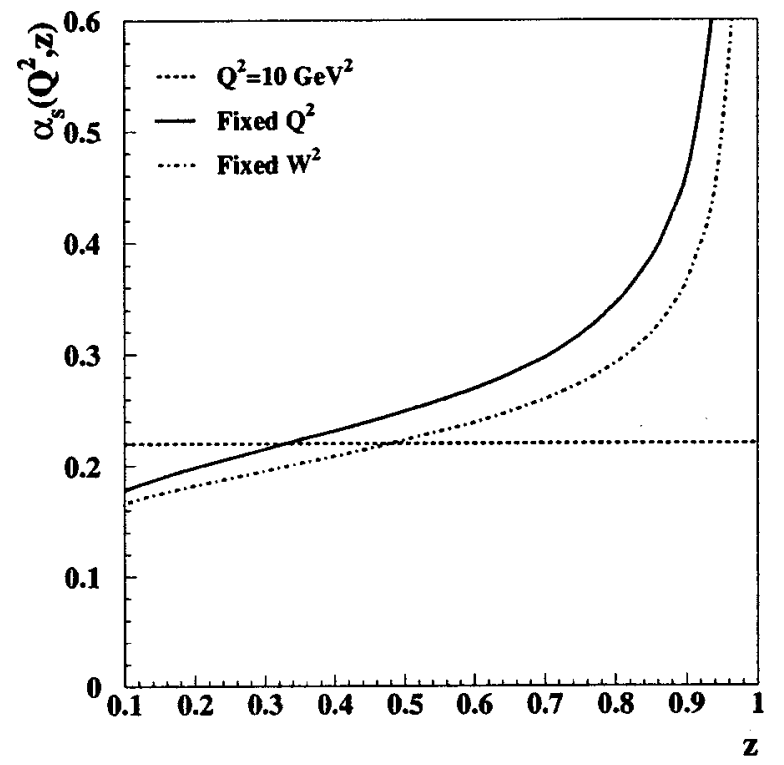

FIG. 6. $\alpha_{S}$ calculated to NLO for different forms of its argument: at $Q^{2}=10 \mathrm{GeV}^{2}$ (dashes), at $Q^{2} \rightarrow Q^{2}(1-z) / z$ (full line), and $Q^{2} \rightarrow W^{2} x /(1-x)(1-z) / z$ (dot-dashed line). Calculations including LxR in the resonance region use the latter form.

correspond also to large $x$ values. Moreover, the effect of TMC is larger than the one of LxR. We have excluded from our analysis the lowest data point at $Q^{2} \approx 0.4 \mathrm{GeV}^{2}$ because of the high uncertainty in both the pQCD calculation and the subtraction of TMC. Also, the pQCD calculations at $Q^{2}$ $\approx 1 \mathrm{GeV}^{2}$ differ from the ones obtained by using the available set of parametrizations perhaps because the latter are extrapolated well beyond their limit of validity.

Similarly, in polarized scattering the inclusion of TMC and LxR decreases the ratio $R_{\text {pol }}^{L T}$ (Fig. 7, bottom panel). However, in this case these effects are included almost completely within the error bars. We conclude that duality is strongly violated at $Q^{2}<1.7 \mathrm{GeV}^{2}$.

The difference between unpolarized and polarized scattering at low $Q^{2}$ can be attributed e.g. to unmeasured, so far, $Q^{2}$ dependent effects, both in the asymmetry, $A_{1}$, and in $g_{2}$. Furthermore, a full treatment of the $Q^{2}$ dependence would require both a more accurate knowledge of the ratio $R$ in the resonance region, and a simultaneous evaluation of $g_{2}$. The present mismatch between the unpolarized and polarized low $Q^{2}$ behavior might indicate that factorization is broken differently for the two processes, and that the universality of partonic descriptions no longer holds.

In Figs. 8 and 9 we address explicitly the question of the size of the HT corrections. We define them for $F_{2}$ as

$$
\begin{aligned}
H\left(x, Q^{2}\right) & =Q^{2}\left(F_{2}^{\mathrm{res}}\left(x, Q^{2}\right)-F_{2}^{\mathrm{LT}}\right) \\
C_{H T}(x) & =\frac{H\left(x, Q^{2}\right)}{F_{2}^{p Q C D}\left(x / Q^{2}\right)} \\
& \equiv Q^{2} \frac{F_{2}^{\mathrm{res}}\left(x, Q^{2}\right)-F_{2}^{L T}}{F_{2}^{\mathrm{LT}}} .
\end{aligned}
$$
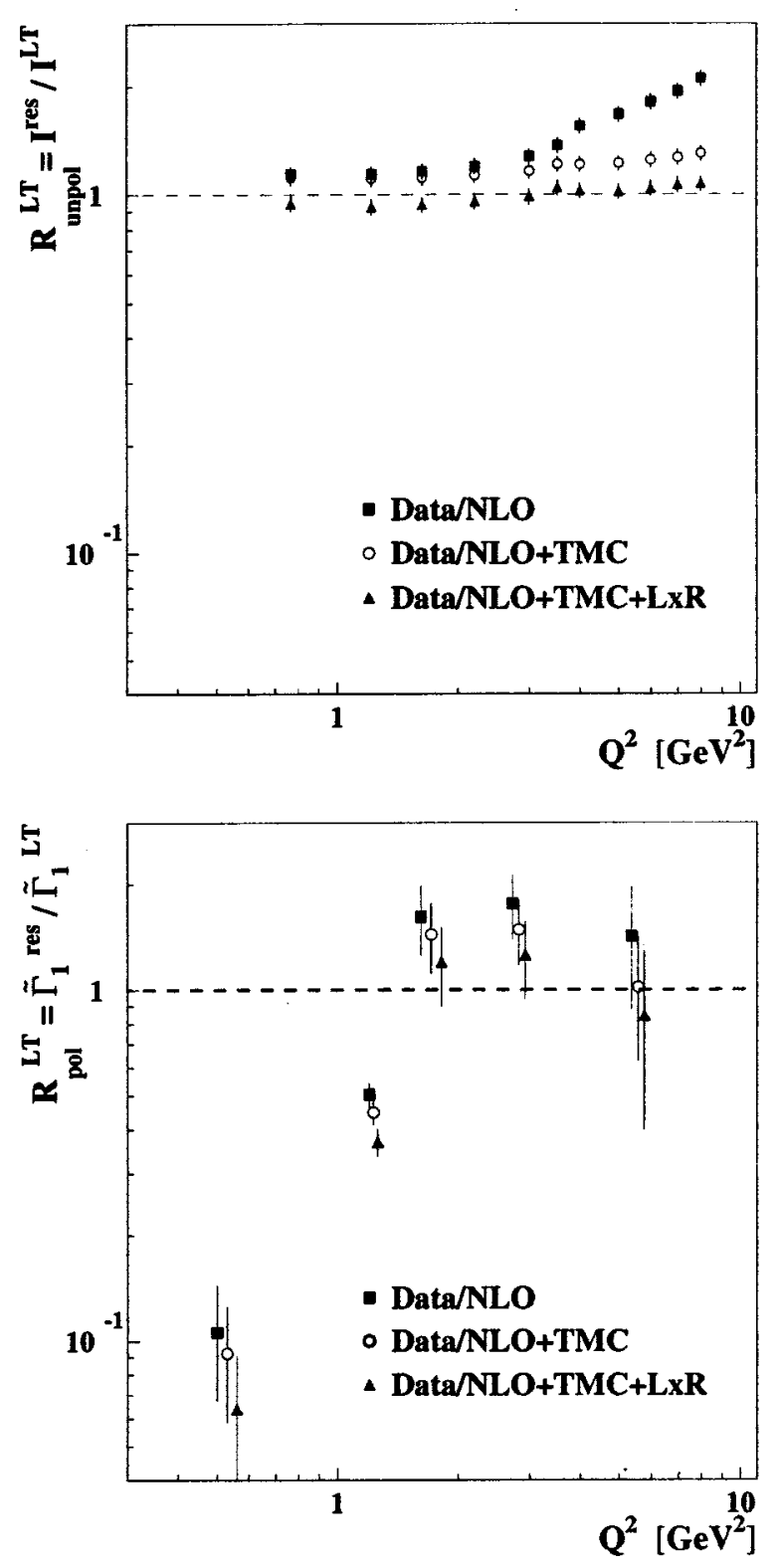

FIG. 7. Ratio between the integrals of the measured structure functions and the calculated ones plotted as a function of $Q^{2}$. The calculation includes one by one the effects of NLO pQCD (squares), TMC (open circles) and LxR (triangles). The top panel refers to the unpolarized case, while the bottom panel to the polarized one.

A similar expression is assumed for $g_{1} \cdot C_{H T}$ is the so-called factorized form obtained by assuming that the $Q^{2}$ dependences of the LT and of the HT parts are similar and therefore they cancel out in the ratio. Although the anomalous dimensions of the HT part could in principle be different, such a discrepancy has not been found so far in accurate analyses of DIS data. The HT coefficient, $C_{H T}$ has been evaluated for the three cases listed also in Fig. 7, namely with respect to the NLO pQCD calculation, to NLO+TMC and to $\mathrm{NLO}+\mathrm{TMC}+\mathrm{LxR}$. The values of $1+C_{H T} / Q^{2}$ are plotted in Fig. 8 (upper panel) as a function of the average value of $x$ for each spectrum. One can see that the NLO 

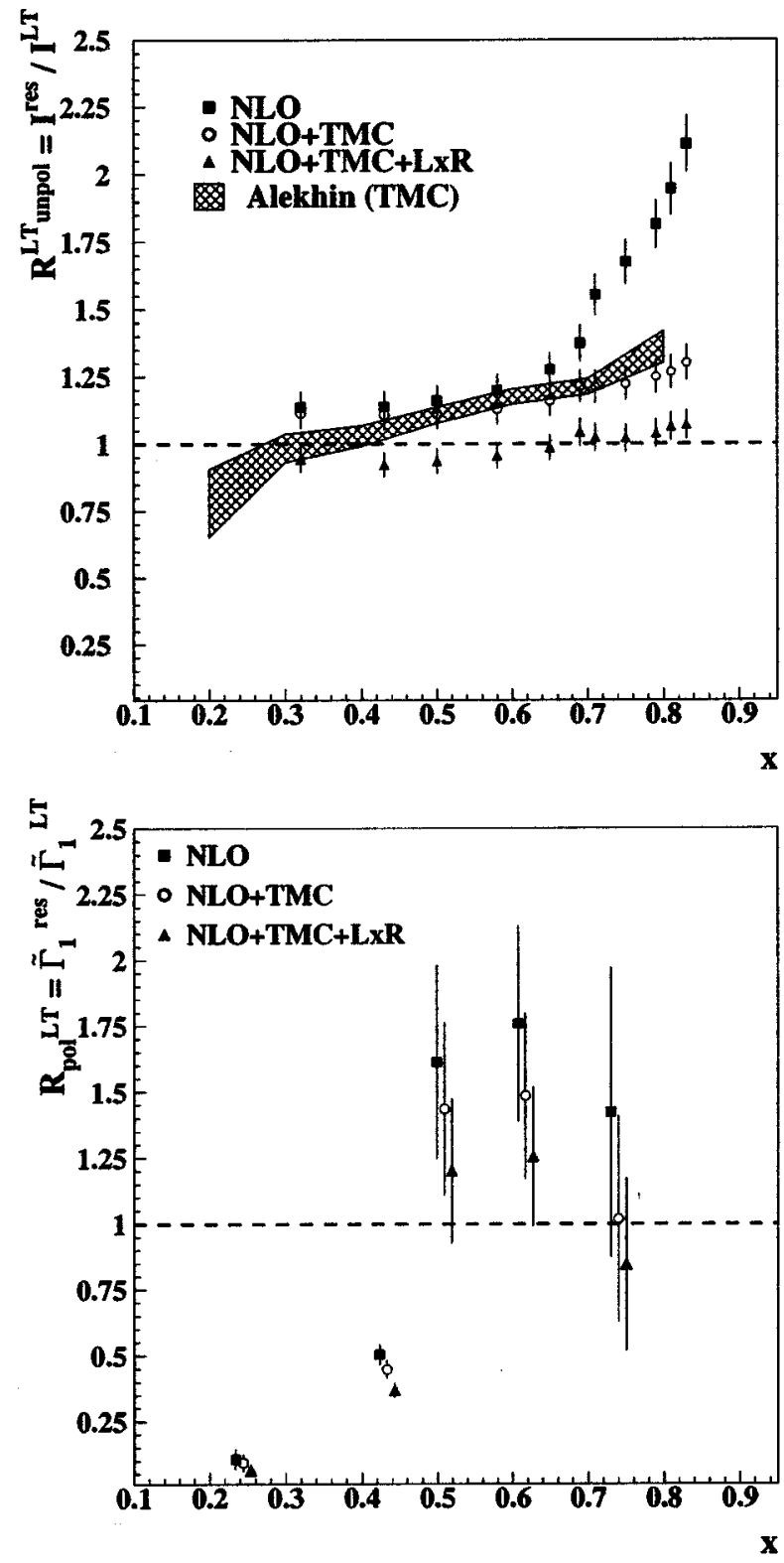

FIG. 8. HT coefficients extracted in the resonance region according to Eq. (24a). Shown in the figure is the quantity 1 $+C_{H T}(x) / Q^{2}$. The top panel refers to the unpolarized case, where we show the HT term obtained by considering only the NLO calculation (squares); the effect of subtracting TMC (open circles); and the effects of subtracting both TMC and LxR (triangles). We show for comparison the values obtained from the coefficient $H$ obtained in Ref. [32] using DIS data and including the effect of TMC. The bottom panel refers to the HT coefficient in the polarized case. Notations are the same as in the upper panel.

$+\mathrm{TMC}+\mathrm{LxR}$ analysis yields very small values for $C_{H T}$ in the whole range of $x$. Furthermore, the extracted values are consistent with the ones obtained in Ref. [6] using a different method, however the present extraction method gives more accurate results. Because of the increased precision of our analysis, we are able to disentangle the different effects from both TMC and LxR.

In the polarized case (Fig. 8, lower panel) the HTs are small within the given precision, for $Q^{2}>1.7 \mathrm{GeV}^{2}$, but they
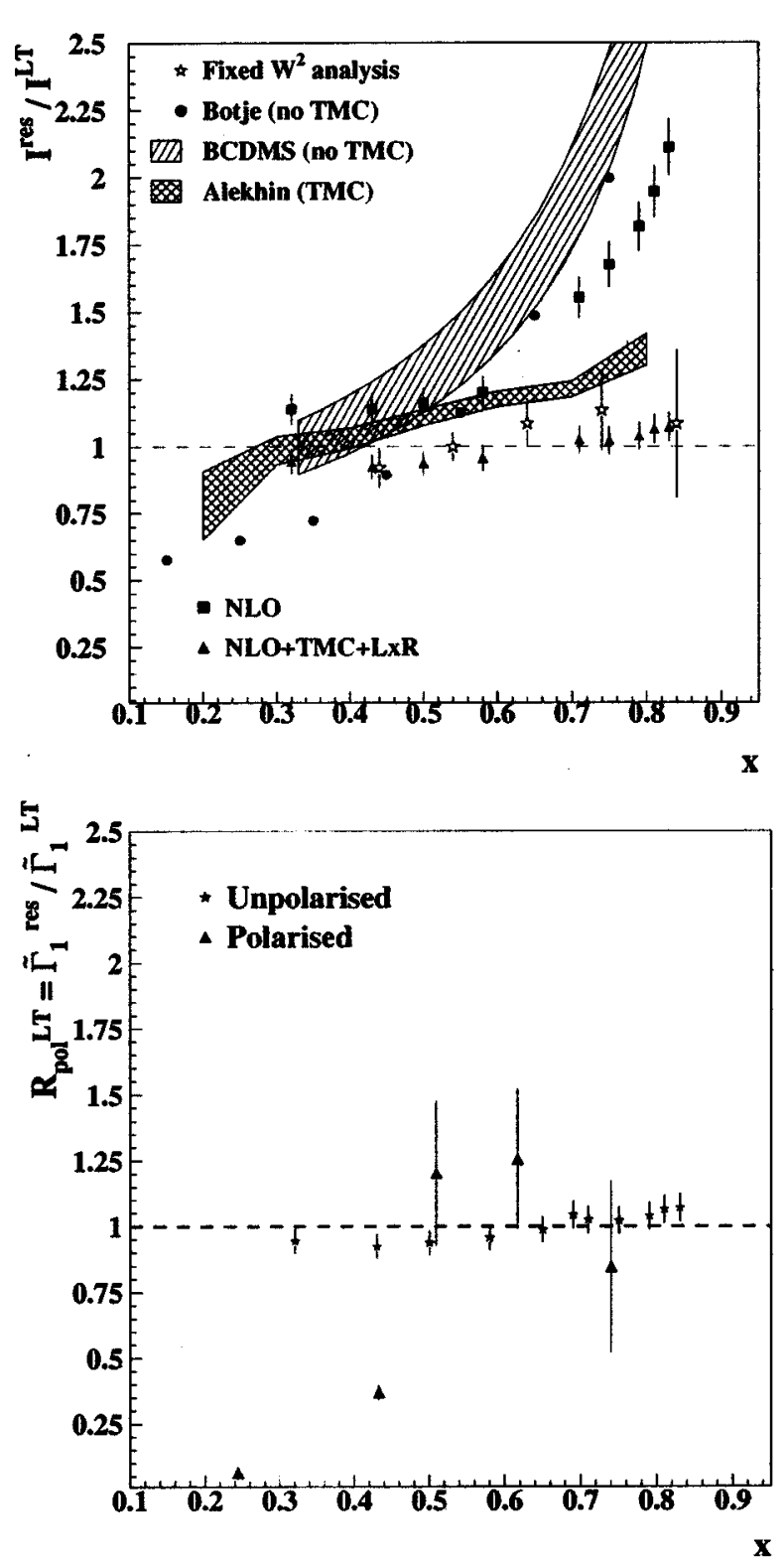

FIG. 9. Comparison of the HT coefficient displayed in Fig. 8, with other extractions (upper panel). The triangles and squares are the same as in Fig. 8 and they represent our determination in the resonance region. Our results are compared with extractions using DIS data only. The striped hatched area corresponds to the early extraction of Ref. [41]. The full dots are the central values of the extractions in Refs. [42] and [43]. These are compared with the more recent extraction of Ref. [32] which includes also TMC. Results obtained in the resonance region, in the fixed $W^{2}$ analysis of Ref. [6] are also shown (stars). In the bottom panel we show the comparison between the HTs in the resonance region, in the polarized (triangles) and in the unpolarized (stars) cases.

appear to drop dramatically below zero for lower $Q^{2}$ values. The inclusion of TMC and LxR renders these terms consistent with zero at the larger $Q^{2}$ values, but it does not modify substantially their behavior at lower $Q^{2}$. It should also be noticed that, by parametrizing the structure functions as in Eqs. (18) and (24a), we are assuming that all of the nonper- 
turbative (np) contributions are included in $\mathcal{O}\left(1 / Q^{2}\right)$ twist-4 terms. These are in fact the largest type of deviations from a pQCD behavior, to be expected at $Q^{2}$ values of the order of few GeV. Only from accurate analyses using a larger number of more precise data would one be able to distinguish among different np behaviors. From a comparison with Fig. 5, that includes some of these extra np behaviors through the usage of phenomenological parametrizations, one can see, however, that their effect seems not to be large.

In Fig. 9 (upper panel) we compare our results in the unpolarized case to other current extractions of the same quantity. These are (i) the extractions from DIS data, performed with the cut $W^{2}>10 \mathrm{GeV}^{2}$ [41-43]; (ii) the recent DIS evaluation by Alekhin [32] using a cut on $W^{2}$ $>4 \mathrm{GeV}^{2}$, and including both TMC and NNLO; (iii) the results obtained within a fixed $W^{2}$ framework in Ref. [6], including both TMC and LxR. We notice that results obtained in Ref. [44] in the deep inelastic region also including both TMC and LxR yield small HT coefficients, consistent with the ones found in Ref. [6]. However, while most of the suppression of the HT in the resonance region is attributed to TMC, in [44] the contribution of TMC is small and the suppression is dominated by LxR. In other words, the $Q^{2}$ behavior in the DIS and resonance regions seems to be dominated by different effects. In Fig. 9 (lower panel) we compare the HT coefficients in the unpolarized and polarized case. One can notice a considerable discrepancy at $Q^{2} \leqslant 1.7 \mathrm{GeV}^{2}$.

Our detailed extraction of both the $Q^{2}$ dependence and the HTs in the resonance region establishes a background for understanding the transition between partonic and hadronic degrees of freedom. In particular, we seem to be detecting a region where the twist expansion breaks down, and at the same time, the data seem to be still far from the $Q^{2} \rightarrow 0$ limit, where theoretical predictions can be made [45]. This breakpoint is marked, for instance, by the discrepancy between polarized and unpolarized scattering at $Q^{2} \leq 1.7 \mathrm{GeV}^{2}$. More studies addressing this region will be pursued in the future, some of which are also mentioned in [6,46]. In particular, a breakdown of the twist expansion can be interpreted in terms of the dominance of multi-parton configurations over single parton contributions in the scattering process. In order to confirm this picture it will be necessary to both extend the studies of the twist expansion, including the possible $Q^{2}$ dependence of the HT coefficients and terms of order $\mathcal{O}\left(1 / Q^{4}\right)$, and to perform duality studies in semi-inclusive experiments. Finally, constituent quark models predict a change in sign in the asymmetry, $A_{1}$, that is expected to become negative at $Q^{2} \leqslant 0.5 \mathrm{GeV}^{2}$, due to the dominance of electric multipole excitations over the magnetic ones $[47,48]$. The onset of this mechanism might explain the departure from unity of the ratios shown in Figs. 8(b) and 9(b). A number of scenarios were studied [29], that consider $S U(6)$ quark parton model breaking effects preserving duality in polarized scattering. In Ref. [29], however, predictions are made only for the larger $Q^{2}$ behavior of the data, whereas the question of duality violations is not addressed explicitly.

\section{CONCLUSIONS}

In summary, we presented a study of parton-hadron duality in both unpolarized and polarized scattering. The latter was obtained by using the first experimental information in the resonance region for the polarized structure function of the proton $g_{1}^{\mathrm{p}}(x)$, for $Q^{2}$ values larger than $1.7 \mathrm{GeV}^{2}$. Parton-hadron duality was analyzed within a QCD context. A pQCD NLO analysis including target mass corrections and large $x$ resummation effects was extended to the integrals of both unpolarized and polarized structure functions in the resonance region. Within our context, duality is satisfied if the $\mathrm{pQCD}$ calculations agree with the data, modulo higher twist contributions consistent with the twist expansion. Although the latter are found to be very small for the unpolarized structure function, we do not conclude that partonhadron duality holds straightforwardly. On the contrary, our findings seem to unveil a richer $Q^{2}$ dynamics both at $x \rightarrow 1$ and at small $x$. This observation is substantiated by the fact that duality holds when comparing data in the resonance region with phenomenological fits which contain some additional "nonconventional" $Q^{2}$ dependence, beyond that predicted by the twist expansion. Most importantly, the coefficient of the HTs extracted using data in the resonance region only, is smaller, and therefore not consistent with the one extracted in the DIS region. Finally, while the size of the HT contributions is comparable in both polarized and unpolarized scattering at larger $x$ and $Q^{2}$ values, at low $x$ and $Q^{2}$ we find large negative nonperturbative contributions only in the polarized case.

\section{ACKNOWLEDGMENTS}

We are indebted to S. Alekhin for discussions and for communications on his calculations prior to publication. We thank W. Melnitchouk and O. Rondon for discussions. S.L. thanks the Gruppo III of INFN at the Laboratori Nazionali di Frascati where this paper was completed, for their warm hospitality, and for the many lively discussions. This work was completed under the U.S. Department of Energy grant no. DE-FG02-01ER41200.
[1] E.D. Bloom and F.J. Gilman, Phys. Rev. Lett. 25, 1140 (1970); Phys. Rev. D 4, 2901 (1971).

[2] M. Gockeler et al., Phys. Rev. D 53, 2317 (1996); LHPC Collaboration and TXL Collaboration, D. Dolgov et al., ibid. 66, 034506 (2002); W. Detmold, W. Melnitchouk, and A.W. Thomas, ibid. 66, 054501 (2002).
[3] M. Battaglia et al., hep-ph/0304132.

[4] A. De Rujula, H. Georgi, and H.D. Politzer, Phys. Lett. 64B, 428 (1976); Ann. Phys. (N.Y.) 103, 315 (1977).

[5] I. Niculescu et al., Phys. Rev. D 60, 094001 (1999); S. Liuti, Nucl. Phys. B (Proc. Suppl.) 74, 380 (1999); X. Ji and P. Unrau, Phys. Rev. D 52, 72 (1995); G. Ricco et al., Phys. Rev. 
C 57, 356 (1998).

[6] S. Liuti, R. Ent, C.E. Keppel, and I. Niculescu, Phys. Rev. Lett. 89, 162001 (2002).

[7] I. Niculescu et al., Phys. Rev. Lett. 85, 1186 (2000).

[8] HERMES Collaboration, A. Airapetian et al., Phys. Rev. Lett. 90, 092002 (2003).

[9] J. Edelmann, G. Piller, N. Kaiser, and W. Weise, Nucl. Phys. A665, 125 (2000).

[10] E143 Collaboration, K. Abe et al., Phys. Rev. D 58, 112003 (1998).

[11] HERMES Collaboration, A. Airapetian et al., Eur. Phys. J. C 26, 527 (2003).

[12] S.J. Brodsky, hep-ph/0006310.

[13] O. Nachtmann, Nucl. Phys. B63, 237 (1973).

[14] A. Szczurek and V. Uleshchenko, Eur. Phys. J. C 12, 663 (2000)

[15] A. Bodek and U.K. Yang, Nucl. Phys. B (Proc. Suppl.) 112, 70 (2002)

[16] H. Georgi and H.D. Politzer, Phys. Rev. D 14, 1829 (1976).

[17] J.L. Miramontes and J. Sanchez Guillen, Z. Phys. C 41, 247 (1988).

[18] C.S. Armstrong, R. Ent, C.E. Keppel, S. Liuti, G. Niculescu, and I. Niculescu, Phys. Rev. D 63, 094008 (2001).

[19] H. Burkhardt and W.N. Cottingham, Ann. Phys. (N.Y.) 56, 453 (1970).

[20] L.W. Whitlow et al., Phys. Lett. B 282, 475 (1992).

[21] A. Fantoni, in Proceedings of the XL International Winter Meeting on Nuclear Physics, Bormio, Italy, 2002, edited by I. Iori and A. Moroni; A. Fantoni, Eur. Phys. J. A 17, 385 (2003); A. Fantoni et al., talk presented at 11th International Workshop On Deep Inelastic Scattering (DIS 2003), St. Petersburg, Russia, 2003.

[22] L.W. Whitlow et al., Phys. Lett. B 250, 193 (1990).

[23] E155 Collaboration, P.L. Anthony et al., Phys. Lett. B 493, 19 (2000)
[24] A.D. Martin, R.G. Roberts, and W.J. Stirling, Eur. Phys. J. C 14, 133 (2000).

[25] H.L. Lai et al., Eur. Phys. J. C 12, 375 (2000).

[26] M. Gluck, E. Reya, and A. Vogt, Z. Phys. C 67, 433 (1995).

[27] M. Gluck, E. Reya, and A. Vogt, Eur. Phys. J. C 5, 461 (1998).

[28] L.V. Gribov and L.N. Lipatov, Yad. Fiz. 20, 181 (1975); G. Altarelli and G. Parisi, Nucl. Phys. B126, 298 (1977); Yu.L. Dokshitzer, Sov. Phys. JETP 46, 641 (1977).

[29] F.E. Close and W. Melnitchouk, Phys. Rev. C 68, 035210 (2003).

[30] H. Abramowicz and A. Levy, hep-ph/9712415.

[31] NMC Collaboration, P. Amaudruz et al., Phys. Lett. B 364, 107 (1995).

[32] (a) S.I. Alekhin, Phys. Rev. D 63, 094022 (2001); 68, 014002 (2003); J. High Energy Phys. 02, 015 (2003); (b) hep-ph/0212370.

[33] M.R. Pennington, Rep. Prog. Phys. 46, 393 (1983).

[34] S. Alekhin, Eur. Phys. J. C 10, 395 (1999).

[35] W.R. Frazer and J.F. Gunion, Phys. Rev. Lett. 45, 1138 (1980).

[36] I. Niculescu, C. Keppel, S. Liuti, and G. Niculescu, Phys. Rev. D 60, 094001 (1999).

[37] S. J. Brodsky and G. P. Lepage, "Perturbative Quantum Chromodynamics," SLAC-PUB-2447.

[38] D. Amati et al., Nucl. Phys. B173, 429 (1980).

[39] R.G. Roberts, Eur. Phys. J. C 10, 697 (1999).

[40] M.R. Pennington and G.G. Ross, Phys. Lett. 102B, 167 (1981).

[41] M. Virchaux and A. Milsztajn, Phys. Lett. B 274, 221 (1992).

[42] A.D. Martin, R.G. Roberts, W.J. Stirling, and R.S. Thorne, Phys. Lett. B 443, 301 (1998).

[43] M. Botje, Eur. Phys. J. C 14, 285 (2000).

[44] S. Schaefer, A. Schafer, and M. Stratmann, Phys. Lett. B 514, 284 (2001).

[45] X.D. Ji and J. Osborne, J. Phys. G 27, 127 (2001).

[46] S. Liuti, Eur. Phys. J. A 17, 385 (2003).

[47] F.E. Close, H. Osborn, and A.M. Thomson, Nucl. Phys. B77, 281 (1974).

[48] V.D. Burkert and B.L. Ioffe, Phys. Lett. B 296, 223 (1992). 